\title{
Country-Specific vs. Common Birthweight-for-Gestational Age References to Identify Small for Gestational Age Infants Born at 24-28 weeks: An International Study
}

Martin, Lisa J ; Sjörs, Gunnar ; Reichman, Brian ; Darlow, Brian A ; Morisaki, Naho ; Modi, Neena ; Bassler, Dirk ; Mirea, Lucia ; Adams, Mark ; Kusuda, Satoshi ; Lui, Kei ; Feliciano, Laura San ;

Håkansson, Stellan ; Isayama, Tetsuya ; Mori, Rintaro ; Vento, Max ; Lee, Shoo K ; Shah, Prakesh S

\begin{abstract}
BACKGROUND Controversy exists as to whether birthweight-for-gestational age references used to classify infants as small for gestational age (SGA) should be country specific or based on an international (common) standard. We examined whether different birthweight-for-gestational age references affected the association of SGA with adverse outcomes among very preterm neonates. METHODS Singleton infants $(\mathrm{n}=23788)$ of $24(0)-28(6)$ weeks' gestational age in nine high-resource countries were classified as SGA ( $<10$ th centile) using common and country-specific references based on birthweight and estimated fetal weight (EFW). For each reference, the adjusted relative risk (aRR) for the association of SGA with composite outcome of mortality or major morbidity was estimated. RESULTS The percentage of infants classified as SGA differed slightly for common compared with country specific for birthweight references $[9.9 \%(95 \%$ CI 9.5, 10.2) vs. $11.1 \%(95 \%$ CI 10.7, 11.5)] and for EFW references $[28.6 \%(95 \%$ CI $28.0,29.2)$ vs. $24.6 \%(95 \%$ CI $24.1,25.2)]$. The association of SGA with the composite outcome was similar when using common or country-specific references for the total sample for birthweight [aRRs 1.47 $(95 \%$ CI 1.43, 1.51) and $1.48(95 \%$ CI 1.44, 1.53) respectively] and for EFW references [aRRs $1.35(95 \%$ CI 1.31, 1.38) and $1.39(95 \%$ CI 1.35, 1.43) respectively]. CONCLUSION Small for gestational age is associated with higher mortality and morbidity in infants born $<29$ weeks' gestational age. Although common and country-specific birthweight/EFW references identified slightly different proportions of SGA infants, the risk of the composite outcome was comparable.
\end{abstract}

DOI: https://doi.org/10.1111/ppe.12298

Posted at the Zurich Open Repository and Archive, University of Zurich ZORA URL: https://doi.org/10.5167/uzh-124547

Journal Article

Accepted Version

Originally published at:

Martin, Lisa J; Sjörs, Gunnar; Reichman, Brian; Darlow, Brian A; Morisaki, Naho; Modi, Neena; Bassler, Dirk; Mirea, Lucia; Adams, Mark; Kusuda, Satoshi; Lui, Kei; Feliciano, Laura San; Håkansson, Stellan; Isayama, Tetsuya; Mori, Rintaro; Vento, Max; Lee, Shoo K; Shah, Prakesh S (2016). Country-Specific vs. Common Birthweight-for-Gestational Age References to Identify Small for Gestational Age Infants Born at 24-28 weeks: An International Study. Paediatric and Perinatal Epidemiology:online.

DOI: https://doi.org/10.1111/ppe.12298 


\title{
Country-Specific vs. Common Birthweight-for-Gestational Age References to Identify Small for Gestational Age Infants Born at 24-28 weeks: An International Study
}

Lisa J. Martin, ${ }^{a}$ Gunnar Sjörs, ${ }^{\mathrm{b}}$ Brian Reichman, ${ }^{\mathrm{c}}$ Brian A. Darlow, ${ }^{\mathrm{d}}$ Naho Morisaki, ${ }^{\mathrm{e}}$ Neena Modi, ${ }^{\mathrm{f}}$ Dirk Bassler, ${ }^{\mathrm{g}}$ Lucia Mirea, ${ }^{\mathrm{a}}$ Mark Adams, ${ }^{g}$ Satoshi Kusuda, ${ }^{\mathrm{h}}$ Kei Lui, ${ }^{\mathrm{i}}$ Laura San Feliciano, ${ }^{\mathrm{j}}$ Stellan Håkansson, ${ }^{\mathrm{k}}$ Tetsuya Isayama, ${ }^{\text {a }}$ Rintaro Mori, ${ }^{1}$ Max Vento, ${ }^{\mathrm{m}}$ Shoo K. Lee, ${ }^{a}$ Prakesh S. Shah, ${ }^{a}$ on behalf of the International Network for Evaluating Outcomes (iNeo) of Neonates Investigators

${ }^{a}$ Maternal-Infant Care Research Centre, Mount Sinai Hospital, Canadian Neonatal Network,Toronto, ON, Canada

${ }^{b}$ Swedish Neonatal Quality Register, Department of Women's and Children's Health, Uppsala University, Uppsala, Sweden

${ }^{c}$ Gertner Institute for Epidemiology and Health Policy Research, Sheba Medical Centre, Israel Neonatal Network, Tel Hashomer, Israel

${ }^{d}$ Australia and New Zealand Neonatal Network, Department of Paediatrics, University of Otago, Christchurch, New Zealand

${ }^{e}$ Department of Social Medicine, National Center for Child Health and Development, Neonatal Research Network Japan, Setagaya-ku, Tokyo, Japan

${ }^{f}$ Neonatal Data Analysis Unit, Section of Neonatal Medicine, Department of Medicine, Imperial College London, UK Neonatal Collaborative, London, UK

${ }^{8}$ Swiss Neonatal Network, Department of Neonatology, University Hospital Zurich, University of Zurich, Zurich, Switzerland

${ }^{h}$ Neonatal Research Network Japan, Maternal and Perinatal Center, Tokyo Women's Medical University, Shinjuku, Tokyo, Japan

${ }^{i}$ Australian and New Zealand Neonatal Network,Royal Hospital for Women,National Perinatal Epidemiology and Statistic Unit,University of New South Wales, Randwick, NSW, Australia

${ }^{j}$ Spanish Neonatal Network, Hospital Universitario de Salamanca, Valencia, Spain

${ }^{k}$ Swedish Neonatal Quality Register, Department of Pediatrics/Neonatal Services, Umeå University Hospital, Umeå, Sweden

${ }^{l}$ Neonatal Research Network Japan, Department of Health Policy, National Center for Child Health and Development, Setagaya-ku, Tokyo, Japan

${ }^{m}$ Spanish Neonatal Network, Health Research Institute La Fe, Valencia, Spain

\begin{abstract}
Background: Controversy exists as to whether birthweight-for-gestational age references used to classify infants as small for gestational age (SGA) should be country specific or based on an international (common) standard. We examined whether different birthweight-for-gestational age references affected the association of SGA with adverse outcomes among very preterm neonates.

Methods: Singleton infants $(n=23788)$ of $24^{0}-28^{6}$ weeks' gestational age in nine high-resource countries were classified as SGA $(<10$ th centile) using common and country-specific references based on birthweight and estimated fetal weight (EFW). For each reference, the adjusted relative risk (aRR) for the association of SGA with composite outcome of mortality or major morbidity was estimated.

Results: The percentage of infants classified as SGA differed slightly for common compared with country specific for birthweight references [9.9\% (95\% CI 9.5, 10.2) vs. 11.1\% (95\% CI 10.7, 11.5)] and for EFW references [28.6\% (95\% CI 28.0,29.2) vs. $24.6 \%$ (95\% CI 24.1, 25.2)]. The association of SGA with the composite outcome was similar when using common or country-specific references for the total sample for birthweight [aRRs 1.47 (95\% CI 1.43, 1.51) and $1.48(95 \%$ CI $1.44,1.53)$ respectively] and for EFW references [aRRs $1.35(95 \%$ CI 1.31, 1.38) and 1.39 (95\% CI $1.35,1.43)$ respectively].

Conclusion: Small for gestational age is associated with higher mortality and morbidity in infants born $<29$ weeks' gestational age. Although common and country-specific birthweight/EFW references identified slightly different proportions of SGA infants, the risk of the composite outcome was comparable.
\end{abstract}

Keywords: Infant, Small for Gestational Age; Infant, Extremely Premature; Neonatal outcomes.

Correspondence:

Prakesh S Shah, Department of Paediatrics, Mount Sinai

Hospital, Rm 19-231F, 600 University Ave, Toronto, ON M5G

1X5, Canada

E-mail: pshah@mtsinai.on.ca.
Fetal growth restriction is a risk factor for perinatal mortality, morbidity, and adverse long-term outcomes. ${ }^{1-3}$ Newborns are classified as small for gestational age (SGA) when birthweight is below the 10th centile of 
birthweight-for-gestational age. Neonates who are SGA are at higher risk of neonatal complications, such as hypoglycaemia, polycythaemia, acute and chronic pulmonary changes, and nutritional and metabolic alterations which can lead to long-term neurodevelopmental and metabolic consequences even at extremely low gestations. Identification of a fetus as IUGR can lead to extra monitoring of fetus, administration of antenatal steroid (far away from actual birth with diminished efficacy), and/or intervention to expedite birth. Similarly, management of a SGA preterm neonate differs with extra monitoring for sugar, blood counts, and investigations to identify cause for SGA along with careful respiratory monitoring and management and altered feeding pattern (slower than usual) and watch for gastrointestinal consequences such as necrotising enterocolitis. The percentage of infants classified as SGA is also a potential case-mix confounding factor in cohort studies evaluating variations in neonatal outcomes across different populations. ${ }^{4}$ However, the choice of birthweight-for-gestational age reference influences the classification of infants as SGA and may impact research findings and clinical practice. ${ }^{5,6}$

Controversy exists regarding the use of a common (international) reference or population-specific birthweight-for-gestational age references to classify infants as SGA. ${ }^{7-9}$ Variation in the observed birthweight-forgestational age exists between countries and different ethnicities. ${ }^{7,10}$ If this variation reflects physiologic differences in birthweight, then it may be appropriate to use country-specific birthweight-for-gestational age references. However, if optimal fetal growth and birth size are similar across populations when maternal health, social, and nutritional conditions are optimal, ${ }^{11}$ then a common birthweight-for-gestational age reference could appropriately be applied.

Another controversy exists regarding the use of birthweight-for-gestational age references based on birthweight or on ultrasound-derived estimates of fetal weight (EFW). Descriptive references, such as the Fenton chart, ${ }^{12}$ are derived from observed birthweights of infants born at various gestational ages and may underdiagnose SGA, ${ }^{13}$ because infants born preterm are more likely to be growth restricted than their counterparts who remain in utero. In contrast, EFW references use estimates of the expected weight of a fetus at a specific gestational age, assuming it had remained in utero until term delivery, and may avoid the bias associated with descriptive birthweight- for-gestational age references. ${ }^{14}$ However, EFW may have substantial measurement error. ${ }^{15}$ There is no consensus as to which type of birthweight-for-gestational age reference is best to identify SGA infants.

The objectives of this study were (i) to estimate the percentage of infants classified as SGA using common and country-specific references based on both birthweight and EFW among very preterm infants in nine countries, (ii) to evaluate the effect of classifying infants as SGA by different birthweight-forgestational age references on the association of SGA with a composite outcome (comprising neonatal mortality or major morbidity), and (iii) to examine whether adjusting for SGA derived from different birthweight-for-gestational age references affects the estimated risk of the composite outcome between populations.

\section{Methods}

\section{Population}

Data on neonates were retrieved from the International Network for Evaluating Outcomes in Neonates (iNeo) database, which contains individual-level data on neonatal characteristics and outcomes from eight national data collection systems (nine countries) between 2007 and 2010. ${ }^{4}$ This included the Australia and New Zealand Neonatal Network (ANZNN), Canadian Neonatal Network (CNN), Israel Neonatal Network (INN), Neonatal Research Network of Japan (NRNJ), Swedish Neonatal Quality Register (SNQ), Neonatal Network of Switzerland (SwissNeoNet), Spanish Neonatal Network (SEN1500), and the United Kingdom Neonatal Collaborative (UKNC).

The population-based sample included 24503 singleton infants born between $24^{0}$ and $28^{6}$ weeks' gestational age without a major congenital malformation. We included neonates $<29$ weeks' gestation as this is the highest risk cohort for adverse outcomes related to preterm birth, and the population coverage for admission to level 3 neonatal units was high enough to avoid selection bias.

Gestational age was determined by the best estimate based on early prenatal ultrasound, last menstrual period, or physical examination at birth, in that order. The following infants were excluded: 118 admitted after 36 weeks post-menstrual age, 19 for missing data for sex, 1 for missing data for birthweight, 6 whose birthweight-for-gestational age was 
$>4$ standard deviations away from the mean, and 571 who were missing data for the composite outcome. The final sample size comprised 23788 (97.1\%) infants.

\section{Classification of SGA}

The birthweight of infants from each collaborator was standardised relative to four different birthweightfor-gestational age references (see below) and expressed as a birthweight $z$-score. ${ }^{16,17}$ Infants with a birthweight $z$-score $<1.28$ (equal to a birthweight below the 10th percentile) were classified as SGA.

\section{Birthweight-gestational age references}

\section{Descriptive (birthweight-based) references}

For all iNeo collaborators, infants were classified as SGA according to the common Fenton birthweightfor-gestational age reference using the Lambda, Mu, and Sigma (LMS) parameters for completed weeks' gestational age provided by Dr. Fenton (personal communication).

Each iNeo collaborator provided a published descriptive birthweight-for-gestational age reference for their population (Table 1). ${ }^{18-24}$ If the birthweightfor-gestational age reference did not provide the mean birthweight and standard deviation by sex and gestational age ${ }^{20,24}$ needed to calculate the birthweight $z$-score, this information was obtained from the network Director (INN, UKCC) from the data source used in the publication. In contrast to the references for other countries, the Japanese and Swedish national birthweight-for-gestational age references excluded infants born by caesarean delivery to reduce potential bias in birthweight from including growth-restricted infants in the reference charts. ${ }^{21,25,26}$ For Japan, we combined the birthweight values for vaginal and caesarean deliveries (obtained by the Director from the same source as the published reference) to create a reference for this study. For Sweden, we used the Canadian reference $^{19}$ because the mean birthweight adjusted for gestational age was similar in Canada and Sweden (926 g and $925 \mathrm{~g}$, respectively).

\section{Estimated fetal weight (EFW)}

For all iNeo collaborators, infants were classified as SGA according to the common EFW reference $\left(\right.$ Salomon $\left.{ }^{14}\right)$. This reference used ultrasound biometric measures to derive EFW with the Hadlock equation ${ }^{27}$ to create fetal growth curves for males and females born from 20 to 36 weeks' gestational age. In addition, we calculated country- and sex-specific EFW references for each collaborator using the global fetal weight reference proposed by Mikolajczyk et al. ${ }^{28}$ In this method, the ratio of the mean birthweight of a specific population at 40 weeks' gestational age to that of the original Hadlock sample $\mathrm{e}^{27}$ is applied to calculate EFW at each gestational age (assumes a constant ratio across gestation). We used birthweight at 40 weeks' gestational age from the country-specific descriptive references and the default SD of 13.2 to create an EFW reference for each collaborator.

\section{Composite outcome}

The primary outcome was a composite of mortality or major morbidity. Because all morbidity outcomes increase risk of mortality, such that a higher mortality rate may result in lower morbidity rates among survivors, we examined a composite outcome to reduce such competing outcomes bias. The composite outcome included mortality due to any cause prior to discharge, major neurological injury defined as grade 3 or 4 intraventricular haemorrhage (IVH) ${ }^{29}$ or periventricular echo densities/echo lucencies, treated retinopathy of prematurity (ROP), ${ }^{30}$ or chronic lung disease defined as oxygen requirement at 36 weeks' post-menstrual age or at discharge. ${ }^{31}$ We also present analyses with mortality alone as the outcome (Table S2).

\section{Statistical analysis}

The percentage of infants classified as SGA and corresponding 95\% confidence intervals (CI) were estimated for the entire cohort and separately for each collaborator according to each of the four birthweightfor-gestational age references.

For each birthweight-for-gestational age reference, the Mantel-Haenszel relative risk for SGA was calculated controlling for gestational age, sex, antenatal corticosteroids, and method of delivery. Adjusted RR was calculated for the composite outcome and for mortality separately using the entire cohort and separately for each iNeo collaborator. The attributable fraction (the proportion of the composite outcome in SGA infants that can be attributed to SGA) was calculated using the adjusted RR by the formula: $(R R-1) / R R$. 


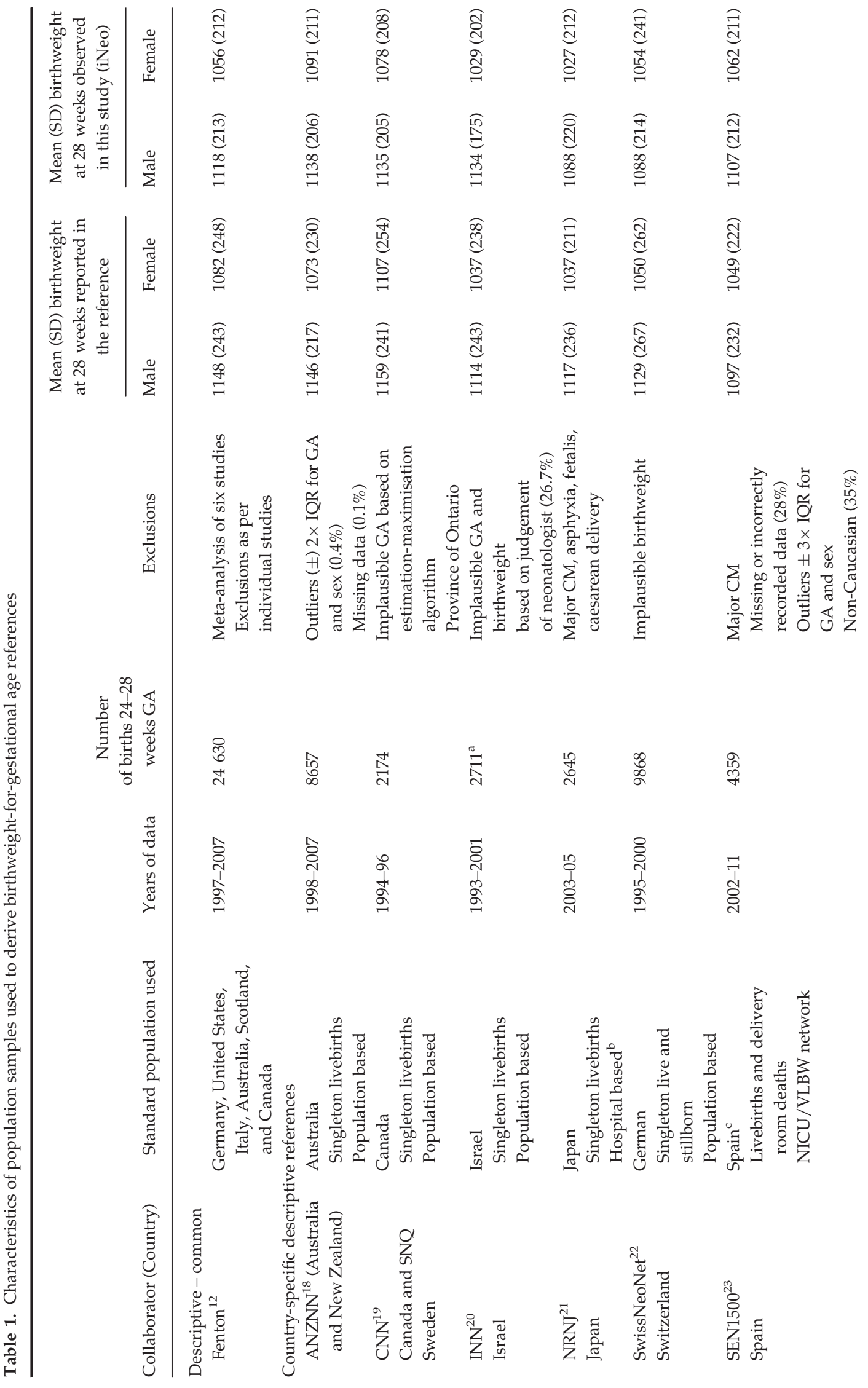

(C) 2016 John Wiley \& Sons Ltd 


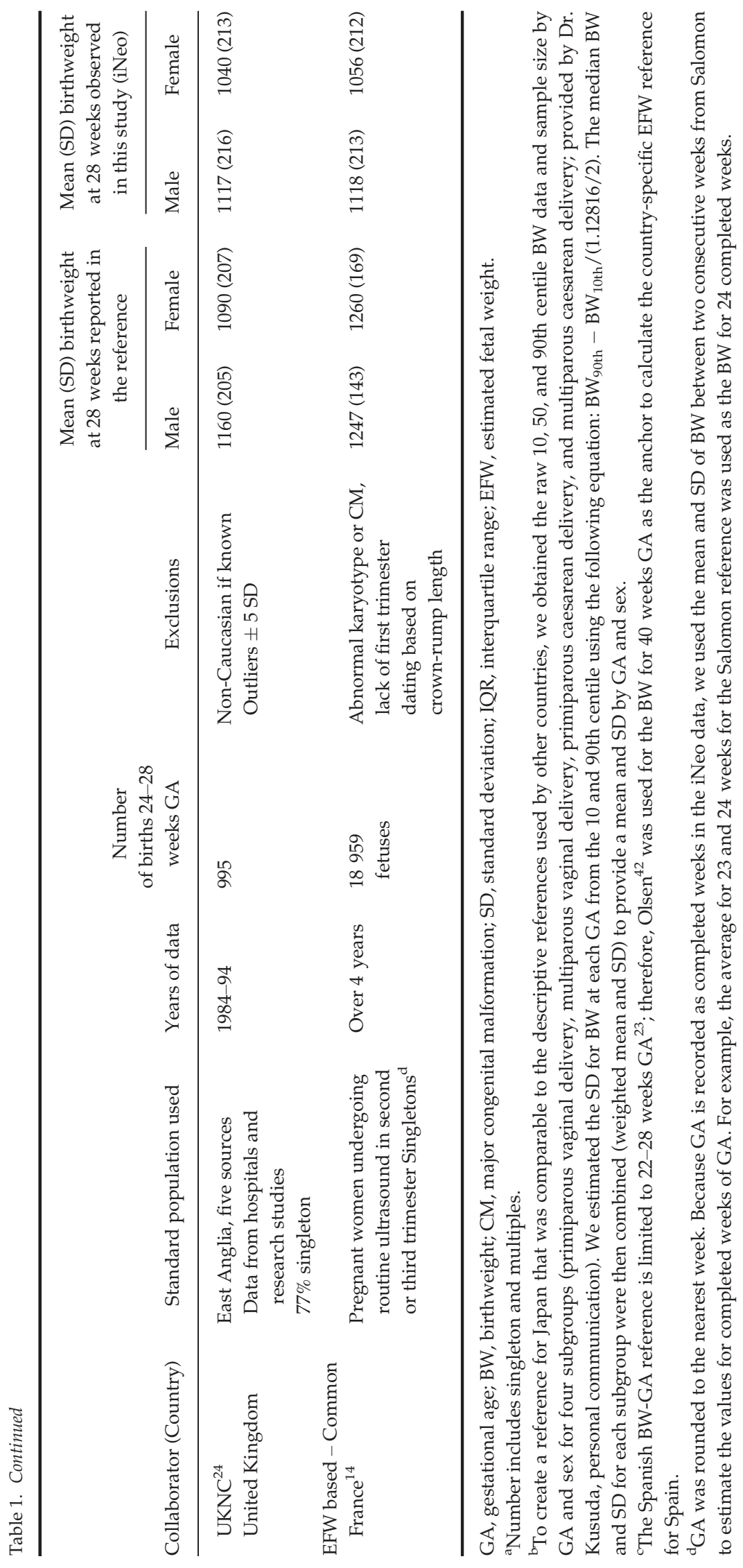


Adjusted RR estimates comparing the composite outcome between each pair of collaborators adjusted for SGA were also estimated for each birthweight-forgestational age reference. The impact of adjusting for SGA derived from different birthweight-for-gestational age references was evaluated by calculating the percentage difference between the pairwise adjusted $R R$ estimates. A difference of $10 \%$ or more in the pairwise adjusted RR estimates was considered a meaningful difference.

All statistical analyses were conducted using SAS version 9.2 with statistical significance evaluated using two-sided test at the $5 \%$ level.

\section{Results}

Within the total cohort, median gestational age was 26 weeks and varied from 26 to 27 weeks between collaborators (Table 2). Mean birthweight varied significantly across collaborators, ranging from $865 \mathrm{~g}$ for NRNJ to $935 \mathrm{~g}$ for ANZNN. The proportion of caesarean deliveries differed significantly between collaborators ranging from $40 \%$ for UKNC to $77 \%$ for SwissNeoNet. The proportion of antenatal corticosteroid use ranged from $47 \%$ for NRNJ to $89 \%$ for ANZNN.

The percentage of infants classified as SGA in the total cohort was $9.9 \%(95 \%$ CI 9.5, 10.2) for the common (Fenton) reference ${ }^{12}$ and $11.1 \%$ (95\% CI 10.7, 11.5) for country-specific birthweight-based references (Table 3). Compared with the birthweight references, the EFW references classified a higher percentage of infants as SGA [28.6\% (95\% CI 28.0, 29.2) for the common EFW and $24.6 \%$ (95\% CI 24.1, 25.2) for country-specific EFW references]. All infants classified as SGA by birthweight-based references were also classified as SGA by EFW-based references.

For all birthweight-for-gestational age references, the percentage of infants classified as SGA varied significantly between collaborators (Pearson chi-square $P<0.0001$; Table 3). For the common birthweight reference (Fenton), SGA ranged from $7.8 \%$ in ANZNN to $15.3 \%$ in SwissNeoNet. Compared with the Fenton reference, the percentage SGA computed using the country-specific birthweight-based references was generally higher. For the common EFW reference, the percentage SGA ranged from $24.6 \%$ in ANZNN to 36.5\% in SwissNeoNet. The percentage of SGA was lower for the country-specific EFW-based references compared with the common EFW-based reference for INN,

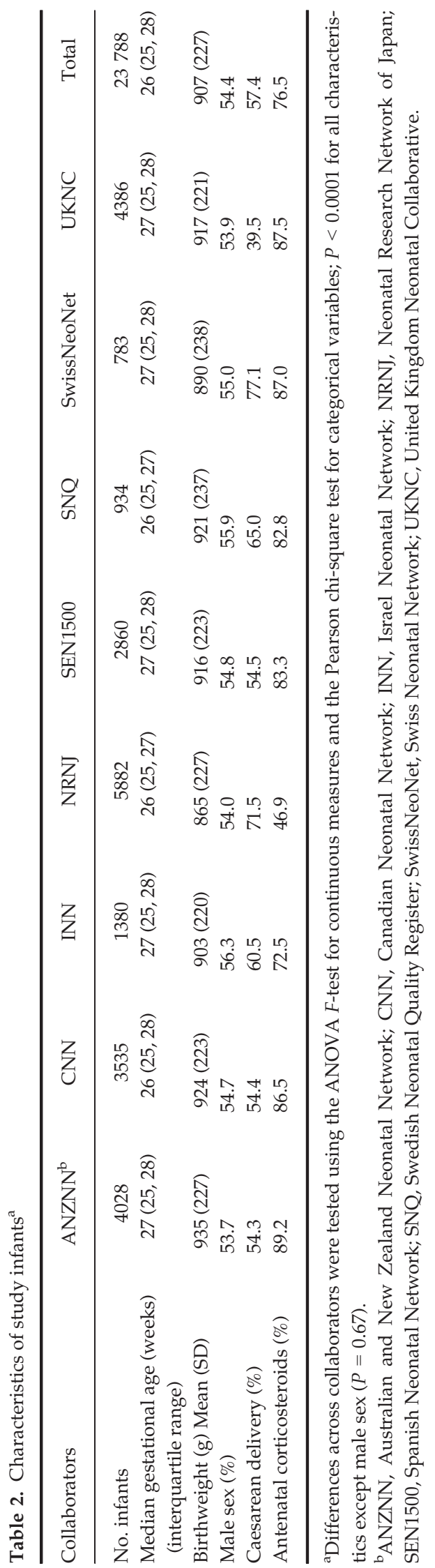

(C) 2016 John Wiley \& Sons Ltd 


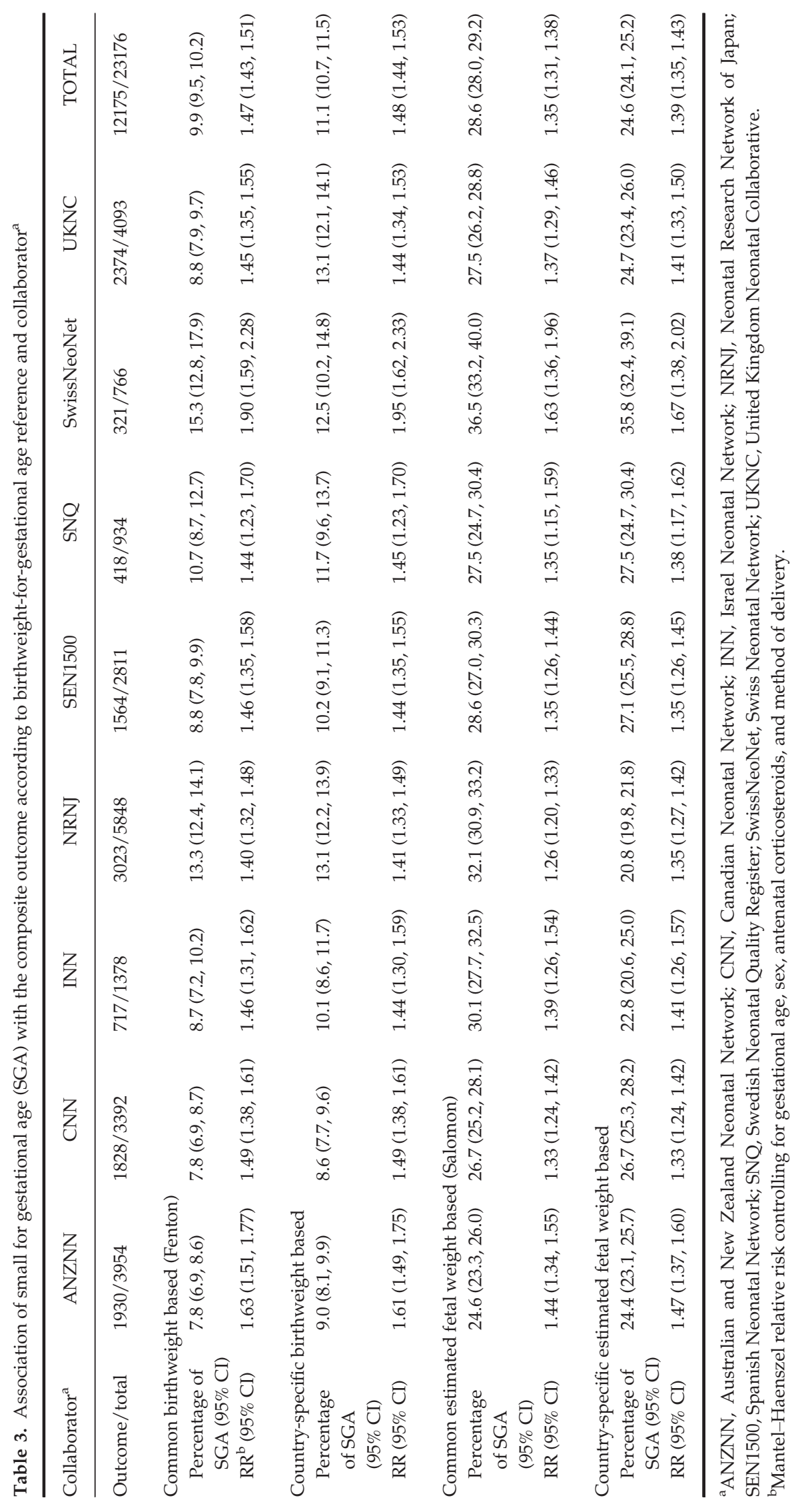


NRNJ, and UKNC. We note that the percentage SGA in the NRNJ was among the highest using all birthweight-for-gestational age references, except for the country-specific EFW reference, where it had the lowest percentage (20.8\%).

Compared with non-SGA infants, SGA infants identified by all birthweight-for-gestational age references were born at a later gestational age, had a higher frequency of caesarean delivery, and were more likely to receive antenatal corticosteroids (Table S1).

In the total cohort, SGA infants had a significantly higher adjusted RR of the composite outcome compared with non-SGA infants for all birthweight-forgestational age references (Table 3 ). The adjusted RRs comparing the composite outcome for SGA to nonSGA infants were similar when SGA was derived from the common or country-specific references. However, the adjusted RRs tended to be lower when SGA was derived from EFW references compared with birthweight references. The adjusted RRs for SGA were fairly similar across collaborators within each reference with slightly higher RR reported for the SwissNeoNet.

Based on the adjusted RR, the fraction of the composite outcome in SGA infants that could be attributed to SGA was $32 \%$ for both common and countryspecific birthweight references, $26 \%$ for common EFW reference, and $28 \%$ for country-specific EFW reference.

The association of SGA with mortality tended to be stronger than with the composite outcome, with adjusted RRs for the whole cohort ranging from 1.66 (95\% CI 1.55, 1.77) for the common EFW reference to 2.11 (95\% CI 1.95, 2.27) for the country-specific birthweight-based references (Table S2).

Sensitivity analyses examined more stringent cut-offs to classify SGA for the common EFW reference. For a cut-off of $-2 \mathrm{SD}$, the percentage of SGA was $16.4 \%(95 \%$ CI $15.9,16.8)$ and the adjusted RR for SGA for the composite outcome was 1.45 (95\% CI 1.41, 1.50). A birthweight $z$-score of $<-2.60$ identified $10 \%$ of infants as SGA and resulted in an adjusted RR of 1.52 (95\% CI 1.48, 1.58). These adjusted RR are similar to those obtained using the BW-based references.

The composite outcome varied significantly between collaborators (overall $P<0.0001$; data not shown). The percent differences in the adjusted RR estimates of the composite outcome for pairwise comparisons between collaborators adjusted for SGA derived from different birthweight-for-gestational age references were all $<4 \%$ and were below the $10 \%$ level determined a priori as a meaningful difference.

\section{Comment}

In this large international cohort of preterm infants born between 24 and 28 weeks' gestational age, the percentage of infants classified as SGA was $10 \%$ and $11 \%$ using common and country-specific birthweightbased references, respectively, compared with $29 \%$ and 25\% when sonographic EFW-based references were used. For each birthweight-for-gestational age reference and for all countries, infants classified as SGA had higher risk for the composite outcome compared with non-SGA infants. Overall and within each country, the magnitude of the adjusted RRs for the composite outcome was similar when SGA status was determined using the common or country-specific birthweight-based references. However, the adjusted RR estimates tended to be lower when SGA was determined from EFW-based references compared with birthweight-based references.

\section{Country-specific vs. common birthweight-for- gestational age references}

The effect of using a common or country/ethnicspecific birthweight-for-gestational age reference in multi-country/ethnic populations has been examined using both birthweight and EFW-based references, but studies were generally limited to stillbirths or mortality outcomes and did not focus specifically on very preterm infants. ${ }^{5,10,28,32,33}$ Common references, generally based on predominantly Caucasian populations in high-income countries, resulted in a higher percentage of SGA infants and a lower OR for the association of SGA with adverse neonatal outcomes compared with country/ ethnic-specific references. ${ }^{5,10,28,32,33}$ This effect is particularly evident for East and South Asian infants, who have a substantially lower average birthweight relative to Caucasian populations. ${ }^{5,32,33}$

In our study, the common (Fenton) birthweightbased reference classified a slightly lower percentage of infants as SGA compared with the country-specific birthweight references in the combined cohort. The Fenton reference was developed with birthweight data from North American and Western European countries, and the similarity of results using Fenton and country-specific descriptive references is not surprising. As expected, countries with the lowest mean birthweight (Japan and Switzerland) had the highest percentage SGA with the common Fenton reference. 
There was minor difference in the percentage of SGA for the common EFW-based reference and the country-specific EFW-based references, with a slight increase in the adjusted RR in SGA neonates for the composite outcome and for mortality. Even though references for country-specific estimation of SGA were derived from each country, a small degree of difference was expected due to some differences in the population due to immigration, when the reference was created because slow and steady change in birthweight has been observed over the years and marginal error due to sampling differences. This may lead to both exaggeration and dilution of differences between country-specific references and common references. More extreme differences have been reported by Mikolajczyk et al., ${ }^{28}$ where SGA decreased from $35 \%$ using the common EFW reference to $11 \%$ for country-specific EFW references, and the odds ratio for SGA compared with non-SGA for perinatal mortality increased substantially. The larger effect of country-specific references in that study likely reflects the fact that average term birthweight in the 24 African, Latin American, and Asian countries was much lower than that of the reference population.

\section{EFW-based compared with birthweight-based birthweight-for-gestational age references}

The risk of developing the composite outcome for infants identified as SGA using the EFW-based references was lower than that for those identified using birthweight-based references. In agreement with this result, a lower OR for SGA was also reported for neonatal mortality in preterm infants when an EFW-based reference was compared with birthweightbased references. ${ }^{34,35}$ The lower risk associated with SGA reflects the fact that the higher birthweight cutoffs of the EFW reference classify a higher number of infants as SGA who represent a less extreme risk group compared with infants classified as appropriate for gestational age. We note that applying a lower cutoff (birthweight $z$-score $<-2.60$ ) for the common EFW reference to classify $10 \%$ of infants as SGA resulted in similar adjusted RR estimates as the Fenton reference.

\section{SGA adds little to prediction of individual outcomes}

SGA infants were at significantly higher risk for the composite outcome and mortality compared with non-SGA infants irrespective of the reference used to classify SGA. Thus, it is an important clinical message that preterm SGA infants need special attention for their care and management. However, SGA status has limited predictive ability for adverse outcomes on an individual level, ${ }^{28,32,36}$ and newer methods, such as determining a sliding scale based on impact on outcomes, or additional markers, such as head circumference or indicators of symmetry of growth, ${ }^{37}$ are needed to better differentiate healthy small babies from pathologically growth-restricted babies.

The choice of birthweight-for-gestational age reference does have implications for neonatologists and perinatologists. EFW-based references (using the 10th percentile cut-off) identify a substantially greater number of SGA infants compared with birthweight-based references; however, the population of infants identified may be at lower risk of adverse outcomes. The use of EFW-based references may thereby increase stress to parents and increase health care costs (as a result of monitoring/intervention more neonates), with marginal benefit, if any, with regards to impact on outcomes. However, this is not to undermine the importance of identifying fetal growth restriction as such infants need to be followed closely.

One striking finding was the similarity of adjusted RR for SGA for adverse outcomes amongst all collaborators of iNeo. This may indicate that the impact of SGA we observed could be used to calculate attributable risk estimates and the fact that we only included extremely low gestation neonates from high-resource countries could support biological plausibility of impact of SGA on mortality and morbidities.

\section{Strengths and limitations}

The large sample size of very preterm infants, the international nature of our cohort derived from eight national data collection systems in nine countries, and our ability to examine a composite adverse outcome (rather than mortality alone) are major strengths of our study. In addition, the accuracy of gestational age and birthweight data is likely high because we examined high-resource countries where a large proportion of women had early ultrasound to determine gestational age. However, the focus on high-resource countries may reduce generalisability of our results. In other contexts, the use of different birthweight for GA references may have greater influence on the risks associated with SGA and/or cross country compar- 
isons of neonatal outcomes. In addition, our study was limited to short-term neonatal outcomes, and the comparison of different birthweight-for-gestational age references may yield different results when examining long-term developmental outcomes. ${ }^{38}$ Although we attempted to harmonise outcome definitions between contributors by generalising classifications, inconsistency in definitions of individual morbidities may have contributed to international variations in the composite outcome. However, the association of SGA with the outcome was similar between contributors, and variation in the definitions does not likely affect our comparison of different birthweight-forgestational age references.

\section{Conclusions}

International (common) growth standards are widely used for children ${ }^{39}$ and have recently been published for infants born $>32$ weeks' gestational age. ${ }^{40}$ In our study of preterm infants of 24-28 weeks' gestational age, the use of common or country-specific birthweight-based references had little influence on the percentage of infants classified as SGA or the association of SGA with neonatal outcome. For studies of neonatal outcomes in preterm infants where SGA classification is important to compare baseline characteristics and/or for use as a confounding variable, we propose to use the Fenton birthweight-based reference for high-resource countries. This reference was created from data from a large number of preterm infants from several countries and removes potential variation associated with independently derived country-specific birthweight-based references. Ongoing work by others examining fetal growth using ultrasound across different populations will provide more information on whether international or countryspecific standards are appropriate for very preterm infants. ${ }^{11,41}$

\section{Acknowledgements}

The Maternal-Infant Care Research Centre is supported by the Canadian Institutes of Health Research and Mount Sinai Hospital, Toronto, Ontario, Canada. We thank all site investigators in nine countries for their support and diligent data collection. We would like to thank Dr Joel Ray, St Michael's Hospital, Toronto, and Dr Liisa Lehtonen, Finland, for their valuable insight in this article.

\section{Funding}

Funding for iNeo has been provided by a Canadian Institutes of Health Research Chair in Reproductive and Child Health Services and Policy Research held by PSS. The Canadian Neonatal Network is funded by financial support from the Canadian Institutes of Health Research and participating centres. The Australian and New Zealand Neonatal Network is predominantly funded by membership contributions from participating centres. The Israel Neonatal Network, Very Low Birthweight Infant Database is partially funded by the Israel Center for Disease Control and the Ministry of Health. The Neonatal Research Network of Japan is partly funded by a Health Labour Sciences Research Grant from the Ministry of Health, Labour and Welfare of Japan. The Swedish Neonatal Quality Register is funded by the Swedish Government (Ministry of Health and Social Affairs) and the body of regional health care providers (County Councils). The United Kingdom Neonatal Collaborative receives no core funding. The Spanish Neonatal Network SEN1500 is funded by the Spanish Neonatal Society (SENeo).

\section{Role of the funders/sponsors}

The funding bodies played no role in the design and conduct of the study; collection, management, analysis, and interpretation of the data; and preparation, review, or approval of the article.

Conflict of interest disclosures: The authors have no conflicts of interest to declare.

\section{References}

1 Kady S, Gardosi J. Perinatal mortality and fetal growth restriction. Best Practice Res Clinical Obstetrics and Gynecology 2004; 18:397-410.

2 Resnik R. Intrauterine growth restriction. Obstettrics and Gynecology 2002; 99:490-496.

3 Gluckman PD, Hanson MA, Copper C, Thornburg KL. Effect of in utero and early life conditions on adult health and disease. New England Journal of Medicine 2008; 35:61-73.

4 Shah PS, Lee SK, Lui K, Sjors G, Mori R, Reichman B, et al. The International Network for Evaluating Outcomes of very low birth weight, very preterm neonates (iNEO): a protocol for collaborative comparisons of international health servides for quality improvement in neonatal care. BMC Pediatrics 2014; 14:110.

5 Katz J, Wu LA, Mullany LC, Coles CL, Lee AC, Kozuki N, et al. Prevalence of small-for-gestational-age and its mortality 
risk varies by choice of birth-weight-for-gestation reference population. PLoS ONE 2014; 9:e92074.

6 Hemming K, Hutton JL, Glinianaia SV, Jarvis SN, Platt MJ. Differences between European birthweight standards: impact on classification of 'small for gestational age'. Developmental Medicine and Childhood Neurology 2006; 48:906-912.

7 Kramer MS. The epidemiology of low birthweight. Nestle Nutition Institute Workshop Series 2013; 74:1-10.

8 Kelly Y, Panico L, Bartley M, Marmot M, Nazroo J, Sacker A. Why does birthweight vary among ethnic groups in the UK? Findings from the Millennium Cohort Study. Journal of Public Health (Oxf) 2009; 31:131-137.

9 Buck Louis GM, Grewal J, Albert PS, Sciscione A, Wing DA, Grobman WA, et al. Racial/ethnic standards for fetal growth: the NICHD Fetal Growth Studies. American Journal of Obstetrics and Gynecology 2015; 213:449.

10 Kierans WJ, Joseph KS, Luo ZC, Platt R, Wilkins R, Kramer MS. Does one size fit all? The case for ethnic-specific standards of fetal growth. BMC Pregnancy and Childbirth 2008; 8:1.

11 Villar J, Papageorghiou AT, Pang R, Ohuma EO, Ismail LC, Barros FC, et al. The likeness of fetal growth and newborn size across non-isolated populations in the INTERGROWTH21 Project: the Fetal Growth Longitudinal Study and Newborn Cross-Sectional Study. Lancet Diabetes and Endocrinology 2014; 2:781-792.

12 Fenton TA, Kim JK. A systematic review and meta-analysis to revise the Fenton growth chart for preterm infants. BMC Pediatrics 2013; 13:59.

13 Burkhardt T, Schaffer L, Zimmermann R, Kurmanavicius J. Newborn weight charts underestimate the incidence of low birthweight in preterm infants. American Journal of Obstetrics and Gynecology 2008; 199:139-6.

14 Salomon LJ, Bernard JP, Ville Y. Estimation of fetal weight: reference range at 20-36 weeks' gestation and comparison with actual birth-weight reference range. Ultrasound in Obstetrics and Gynecology 2007; 29:550-555.

15 Mayer C, Joseph KS. Fetal growth: a review of terms, concepts and issues relevant to obstetrics. Ultrasound in Obstetrics and Gynecology 2013; 41:136-145.

16 Land JA. How should we report on perinatal outcome? Human Reproduction 2006; 21:2638-2639.

17 Fenton TR, Sauve RS. Using the LMS method to calculate z-scores for the Fenton preterm infant growth chart. European Journal of Clinical Nutrition 2007; 61:1380-1385.

18 Dobbins TA, Sullivan EA, Roberts CL, Simpson JM. Australian national birthweight percentiles by sex and gestational age, 1998-2007. Medical Journal of Australia 2012; 197:291-294.

19 Kramer MS, Platt RW, Wen SW, Joseph KS, Allen A, Abrahamowicz $\mathrm{M}$, et al. A new and improved populationbased Canadian reference for birth weight for gestational age. Pediatrics 2001; 108:E35.

20 Dollberg S, Haklai Z, Mimouni FB, Gorfein I, Gordon ES. Birth weight standards in the live-born population in Israel. Israel Medical Association Journal 2005; 7:311-314.

21 Itabashi K, Miura F, Uehara R, Nakamura Y. New Japanese neonatal anthropometric charts for gestational age at birth. Pediatric International 2014; 56:702-708.
22 Voigt M, Fusch C, Olbertz D, Hartmann K, Rochow N, Renken C. Analysis of the Neonatal Collective in the Federal Republic of Germany, 12th Report: presentation of detailed percentiles for the body measurement of newborns. Geburtsh Frauenheilk 2006; 66:956-970.

23 Garcia-Munoz Rodrigo F, Garcia-Alox Perex A, Figueras Aloy J, Saavedra Santana P. New population growth curves in Spanish extremely preterm neonates. Annals of Pediatrics (Barc) 2014; 81:107-114.

24 Cole TJ, Williams AF, Wright CM. Revised birth centiles for weight, length and head circumference in the UK-WHO growth charts. Annals of Human Biology 2011; 38:7-11.

25 Uehara R, Miura F, Itabashi K, Fujimura M, Nakamura Y. Distribution of birth weight for gestational age in Japanese infants delivered by cesarean section. Journal of Epidemiology $2011 ; 21: 217-222$.

26 Niklasson A, Albertsson-Wikland K. Continuous growth reference from 24 th week of gestation to 24 months by gender. BMC Pediatrics 2008; 8:8.

27 Hadlock FP, Harrist RB, Martinez-Poyer J. In utero analysis of fetal growth: a sonographic weight standard. Radiology 1991; 181:129-133.

28 Mikolajczyk RT, Zhang J, Betran AP, Souza JP, Mori R, Gulmezoglu AM, et al. A global reference for fetal-weight and birthweight percentiles. The Lancet 2011; 377:1855-1861.

29 Papile LA, Burstein J, Burstein R, Koffler H. Incidence and evolution of subependymal and intraventricular hemorrhage: a study of infants with birth weights less than 1,500 gm. Journal of Pediatrics 1978; 92:529-534.

30 The International Classification of Retinopathy of Prematurity revisited. Archives of Ophthalmology 2005; 123:991-999.

31 Shennan AT, Dunn MS, Ohlsson A, Lennox K, Hoskins EM. Abnormal pulmonary outcomes in premature infants: prediction from oxygen requirement in the neonatal period. Pediatrics 1988; 82:527-532.

32 Urquia ML, Berger H, Ray JG. Risk of adverse outcomes among infants of immigrant women according to birthweight curves tailored to maternal world region of origin. Canadia Medica Association Journal 2015; 187:E32-E40.

33 Hanley GE, Janssen PA. Ethnicity-specific birthweight distributions improve identification of term newborns at risk for short-term morbidity. American Journal of Obstetrics and Gynecology 2013; 209:428-6.

34 Zaw W, Gagnon R, da SO. The risks of adverse neonatal outcome among preterm small for gestational age infants according to neonatal versus fetal growth standards. Pediatrics 2003; 111:1273-1277.

35 Cooke RW. Conventional birth weight standards obscure fetal growth restriction in preterm infants. Archives of Diseases of Childhood Fetal Neonatal Edition 2007; 92: F189-F192.

36 Ferdynus C, Quantin C, Abrahamowicz M, Burguet A, Sagot P, Gouyon JB. Comparison of the ability of alternative birthweight and fetal weight standards to identify preterm newborns at increased risk of perinatal death. British Journal of Obstetrics and Gynecology 2013; 120:1456-1464. 
37 Guellec I, Marret S, Baud O, Cambonie G, Lapillonne A, Roze JC, et al. Intrauterine growth restriction, head size at birth, and outcome in very preterm infants. Journal of Pediatrics 2015; 167:975-981.

38 Charkaluk ML, Marchand-Martin L, Ego A, Zeitlin J, Arnaud C, Burguet A, et al. The influence of fetal growth reference standards on assessment of cognitive and academic outcomes of very preterm children. Journal of Pediatrics 2012; 161:1053-1058.

39 de Onis M, Onyango A, Borghi E, Siyam A, Blossner M, Lutter C. Worldwide implementation of the WHO Child Growth Standards. Public Health Nutrition 2012; 15: 1603-1610.

40 Villar J, Cheikh IL, Victora CG, Ohuma EO, Bertino E, Altman DG, et al. International standards for newborn weight, length, and head circumference by gestational age and sex: the Newborn Cross-Sectional Study of the INTERGROWTH-21st Project. Lancet 2014; 384:857-868.

41 Merialdi M, Widmer M, Gulmezoglu AM, Abdel-Aleem H, Bega G, Benachi A, et al. WHO multicentre study for the development of growth standards from fetal life to childhood: the fetal component. BMC Pregnancy and Childbirth 2014; 14:157.

42 Olsen IE, Groveman SA, Lawson ML, Clark RH, Zemel BS. New intrauterine growth curves based on United States data. Pediatrics 2010; 125:e214-e224.

\section{Supporting Information}

Additional Supporting Information may be found in the online version of this article at the publisher's web-site:

Table S1. Characteristics of infants classified as SGA/ non-SGA using different birthweight-for-gestational age references.

Table S2. Association of small for gestational age (SGA) with mortality according to birthweight-forgestational reference and collaborator list of contributing neonatal units. 